\title{
Electromagnetic Energy Harvesting Using a Glass Window
}

\author{
Yan S. Gonçalves (D), Ursula C. Resende (D), Ícaro V. Soares (1) \\ Federal Center for Technological Education of Minas Gerais (CEFET-MG) \\ Belo Horizonte, Minas Gerais, Brasil \\ yanoliver16@gmail.com,resendeursula@cefetmg.br,icarocosmos@gmail.com
}

\begin{abstract}
In this work, a new constructive and conceptual model for rectenna, using glass as dielectric substrate, was proposed. This research aims to produce a window for building facades that can harvest the electromagnetic energy available in the environment. For this proposal, a slotted patch antenna was designed with two main objectives: to have a low value of $S_{11}$ parameter and to allow the maximum visibility through the glass. In order to accomplish an efficient radio frequency to direct current conversion, a voltage doubler rectifier was designed and built in the same glass substrate, directly connected to the antenna. The final system is a window composed of eight individual rectennas associated in parallel. For improving the window energy harvesting ability, a metamaterial structure was added to each rectenna element, which acts in order increases the patch antenna gain. Each rectenna was designed by using both Advanced Design System and Computer Simulation Technology softwares and optimized to operate at $2.45 \mathrm{GHz}$. The measured results show that the proposed rectenna model is an efficient solution to improve the total amount of harvested energy, which is enough to power a low consumption load.
\end{abstract}

Index Terms - Electromagnetic energy harvesting, glass dielectric substrate, metamaterial, patch antennas, rectenna.

\section{INTRODUCTION}

The growing demand for cheap and clean energy sources has been motivating the study and development of distinct technologies and devices, which are able to provide different amounts of electrical power. Specifically, to supply energy for small loads, the power from the electromagnetic spectrum can be harvested. This possibility is particularly interesting because this kind of energy is constantly available in the environment and the number of Radio-Frequency (RF) sources is progressively increasing, especially in large urban centers. The efficient electromagnetic energy harvesting can be performed by a rectenna, which is an equipment composed of an antenna and a rectifier circuit [1]-[2].

The recent and fast development of the internet, wireless communication technologies and lowpower electronic devices has led to the Smart Applications (SA) technology expansion and popularization. This technology has been massively applied in the development of smart city solutions, lower power consumption devices, Internet of Things (IoT) and 4G and 5G applications. The main SA technology feature is the use of Wireless Sensor Networks (WSNs). Although theses sensors are low-power loads [3] their use imposes huge challenges in terms of a new, efficient and Brazilian Microwave and Optoelectronics Society-SBMO received 13 Sept 2019; for review 19 Sept 2019 ; accepted 24 Nov 2019 
reliable way for power supply in order to avoid the traditional battery. The RF-based energy harvesting technology is especially suitable to power WSNs because the rectenna can be completely introduced in the distributed hosting sensors structure, reducing its cost, maintenance and environmental impact [4].

Nowadays different researches have been carried out in order to use the rectennas to efficiently pick up the RF energy available in the environment and convert it into DC for either using it directly to supply a low power consumption device or storing it for later use [5]-[6]. However, since the amount of electromagnetic energy available in the environment is very small [4], limited power values can be harvested by the rectenna. Therefore, different technical strategies have been investigated in order to increase the harvested power. Among these strategies can be highlighted the use of several rectenna elements connected in parallel so that the total harvested energy can be increased [7]-[8]. Different types of antennas have been researched for rectenna applications, however, patch antennas are preferred due to their reduced cost, easy construction and compact size. To increase the patch antenna gain and consequently the rectenna ability to harvest RF energy, the use of different dielectric substrates has been investigated [9]. The inclusion of metamaterial (MTM) in the antenna composition has also been studied for increasing its gain and operating band and consequently improving the rectenna performance [10].

In this work, a new rectenna conceptual model and application are proposed. The novelty is the design of a set of rectennas, mounted on a glass substrate, which can be used as buildings windows and simultaneously harvesting electromagnetic energy from the environment. The proposed rectenna is composed of a $2.45 \mathrm{GHz}$ patch antenna and a voltage doubler rectifier circuit, built in the same glass substrate. In order to increase the amount of energy harvested, eight individual rectennas, incorporating MTM cells, were connected in parallel forming a system, denominated Electromagnetic Window (EW). The EW experimental evaluation demonstrated that the collected power is enough to supply low consumption loads, such as WSNs [3].

\section{ANTENNA DESIGN}

The antenna function in a rectenna is to collect as much RF radiation as possible and transfer it to the rectifier circuit. For the EW proposed, it is desirable that the patch antenna radiant element be compact and allow visibility through the window. Then a rigorous project must be carried out in order to gather compactness requirements and high radiation performances. In this work, the antenna was designed separately from the rectifier circuit by using Computer Simulation Technology (CST) software employing the Finite Difference Time Domain (FDTD) technique for the electromagnetic evaluation.

\section{A. Slotted Patch Antenna}

A slotted patch antenna, that is a patch antenna with an opening, as illustrated in Fig. 1 and represented in this work by the acronym PAO, was the geometry choose for the first rectenna Brazilian Microwave and Optoelectronics Society-SBMO received 13 Sept 2019; for review 19 Sept 2019; accepted 24 Nov 2019 $\begin{array}{lllll}\text { Brazilian Society of Electromagnetism-SBMag } & \text { () 2020 SBMO/SBMag } & \text { (cc) BY } & \text { ISSN 2179-1074 }\end{array}$ 
prototype investigated. The antenna radiating element was designed to be fabricated by using a $30 \mu \mathrm{m}$ thickness copper adhesive tape glued on a $2.9 \mathrm{~mm}$ thickness glass dielectric substrate with experimentally characterized relative electric permittivity $\varepsilon_{\mathrm{r}}=7.89$ and loss tangent $\delta=0.0054$ at 2.45 GHz. In order to obtain high gain and values of $S_{11}$ parameter below $-10 \mathrm{~dB}$ at $2.45 \mathrm{GHz}$, the Genetic Algorithm (GA) technique, from CST, was chosen to optimize the geometrical parameters XL, YL, $\mathrm{XA}, \mathrm{YA}, \mathrm{XO}$, and YO, illustrated in Fig. 1. The GA was executed by using an initial population of 32 individuals, mutation rate equal to $60 \%, 30$ iterations and number of solver evaluations equal to 497 . The optimization process results, presented in Table I, were used to fabricate a PAO prototype, which was experimentally evaluated by using the network analyzer Keysight ${ }^{\circledR}$ E5071C. The experimental and numerical results obtained are presented in Fig. 2. As it can be observed, the antenna is resonant at $2.45 \mathrm{GHz}$, however with low gain value, according to the radiation pattern illustrated in Fig 3 . There is a good agreement between numerical and experimental results and the divergences occurred mainly due to glass non-homogeneity and anisotropy and to the manual manufacturing process.

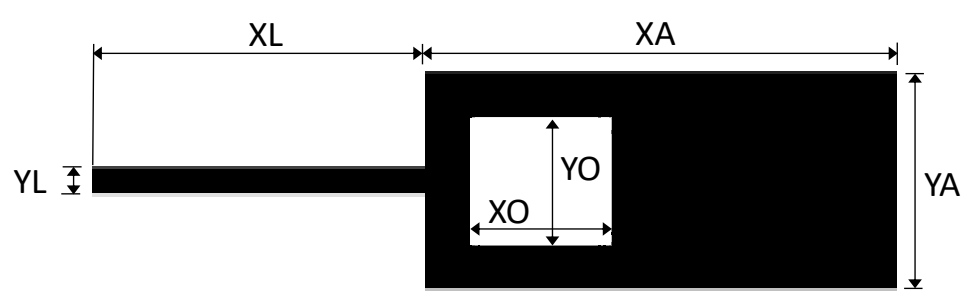

Fig. 1. PAO radiating element.

TABLE I. ANTENNA GEOMETRICAL AND ELECTROMAGNETIC PARAMETERS

\begin{tabular}{ccc}
\hline Antenna parameters & PAO & PAO-MTM \\
\hline XL(mm) & 30.244 & 30.244 \\
YL (mm) & 1.989 & 1.989 \\
XA (mm) & 42.983 & 42.983 \\
YA (mm) & 15.800 & 15.800 \\
XO (mm) & 12.494 & 12.494 \\
YO (mm) & 9.920 & 9.920 \\
XM (mm) & - & 4.558 \\
YM (mm) & - & 4.571 \\
XS = YS1 (mm) & - & 2.642 \\
XD & - & 3.928 \\
YD & - & 2.342 \\
YS2 & - & 5.641 \\
Simulated S 11 (2.45 GHz) $(\mathrm{dB})$ & -15.450 & -15.770 \\
Measured S & -12.300 & -17.580 \\
Gain $(\mathrm{dBi})$ & 2.410 & 4.510 \\
\hline
\end{tabular}




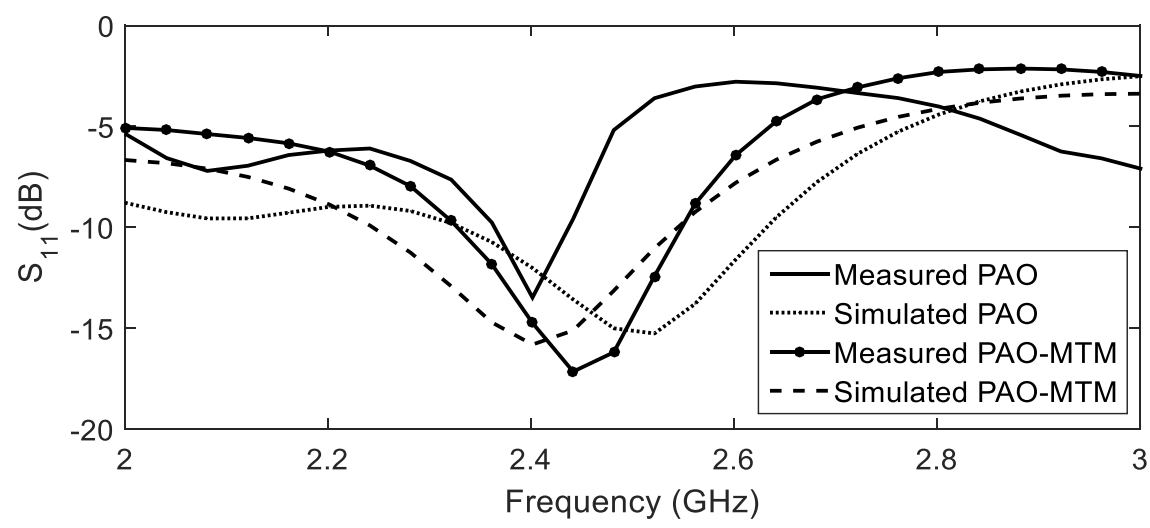

Fig.2. Simulated and measured $S_{11}$ parameter for PAO and PAO-MTM.

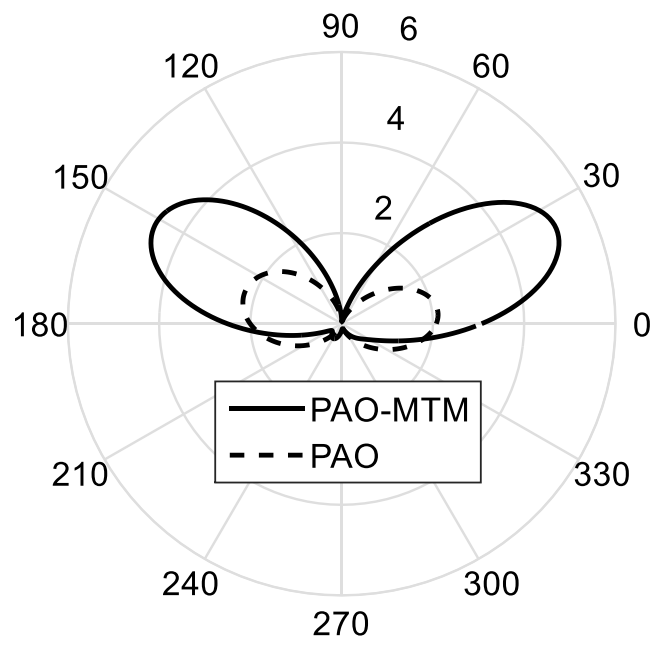

Fig.3. Simulated radiation pattern (dBi) for PAO and PAO-MTM.

\section{B. Slotted Patch Antenna with Metamaterial Cells}

MTM are artificial materials designed with ordinary materials that exists in nature. They are composed with unit cells, arranged in a periodic manner, for which the average size must be much smaller than the wavelength of the electromagnetic field to be modified [11]. Metamaterials properties are not defined only by their material composition but also by their structural organization. This feature allows them to be designed so that they can manipulate electromagnetic waves in order to achieve a desired behavior that go beyond those possible by using only conventional materials. Therefore, due the MTM ability to modify electromagnetic fields its inclusion in the antenna structure has been investigated in order to improve the conventional patch antenna performance in terms of return loss, impedance matching, gain, bandwidth and size reduction [10], [12].

In this work, the inclusion of MTM cells in the antenna structure was investigated in order to reduce its $S_{11}$ parameter value and to enhance its bandwidth and gain. So, a set of square MTM cells were placed near to the unchanged PAO radiating element, as illustrated in Fig. 4. This new antenna geometry is represented in this work by the acronym PAO-MTM. The GA technique, with the same Brazilian Microwave and Optoelectronics Society-SBMO received 13 Sept 2019; for review 19 Sept 2019; accepted 24 Nov 2019 
settings, was again employed, at this time, to adjust the MTM unit cells geometry and arrangement. The obtained results were also presented in Fig. 2, Fig. 3 and Table I. As it can be observed, they meet the established requirements since the antenna gain, bandwidth has increased, and $S_{11}$ parameter has declined slightly. The optimization process results were used to fabricate a PAO-MTM prototype, which was experimentally evaluated and the results obtained, also presented in Fig. 2, show good agreement with the simulated one.

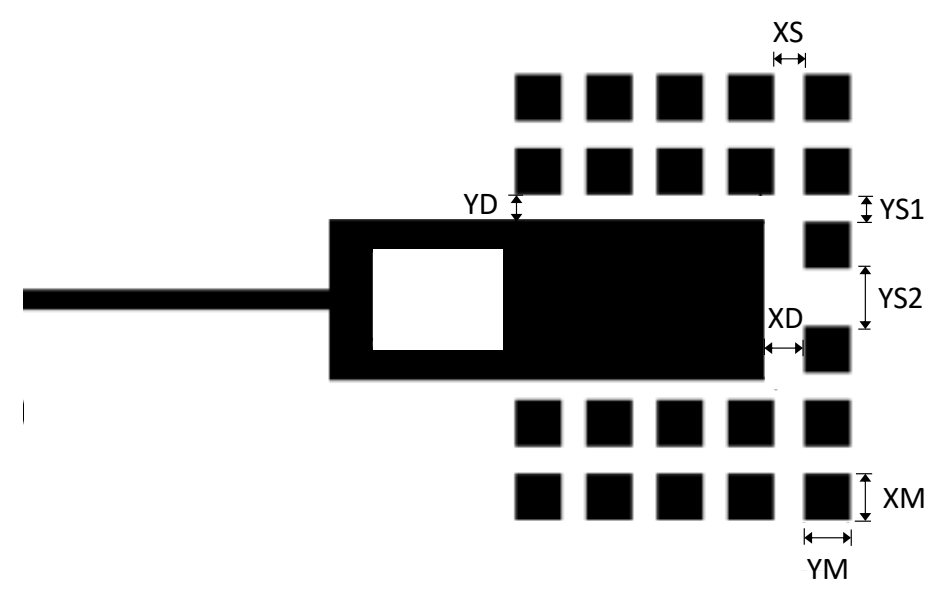

Fig. 4. PAO radiating element with MTM cells

\section{RECTIFIER CIRCUIT DESIGN}

In a rectenna, the rectifier is a nonlinear circuit, which converts a very low input RF energy (less than $-10 \mathrm{dBm}$ ) into DC voltage. The main operational parameter of a rectenna is its efficiency, determined by its losses, which arise during this conversion process. Therefore, in this work, a rectifier with high conversion efficiency under low power input levels was proposed and its topology is shown in Fig. 5. It consists of a voltage doubler circuit composed of a HSMS-2852 microwave Silicon Schottky detector diode pair (D1) and two $100 \mu \mathrm{F}$ capacitors (C1 and C2). The voltage doubler configuration was chosen because its output DC voltage is approximately the double of a single diode and it leads to a simple topology, which is desired for the construction over a glass substrate by using adhesive copper strips as a conductive trace. The HSMS-2852 consists of two diodes connected in series and mounted into a single package, which simplifies the rectifier topology. It was selected because it has a low forward voltage (between 150 and $250 \mathrm{mV}$ ), high cutoff frequency and its SPICE model is available in Advanced Design System (ADS) software. The capacitor C1 acts as a band-pass filter rejecting the undesired harmonics generated by the Schottky diode. The capacitor C2 acts as an output filter to improve DC output voltage level and quality. The value of $\mathrm{C} 2$ has been set according to the internet Wi-Fi commercial router model TL-WR841HP operating cycle. The value of capacitors $\mathrm{C} 1$ and $\mathrm{C} 2$ must be equal in order to ensure high voltage values at the rectifier output. The impedance matching circuit is composed of transmission lines TL1 and TL2 and the stub S1, illustrated in Fig. 5. On the opposite side of the glass, under the rectifier circuit, was positioned a ground plane with length equal to $\mathrm{X} 1+\mathrm{X} 2+\mathrm{X} 3+\mathrm{X} 4+\mathrm{X} 4+\mathrm{XL} / 2$. 
The ADS Harmonic-Balance (HB) simulator and GA technique, adjusted with an initial population of 32 individuals, were used to optimize the rectifier dimensions (X1, X2, X3, X4, X5, Y1 and Y2) illustrated in Fig.5. The optimization process was carried considering a low input power $(-10 \mathrm{dBm})$ with goals of high-efficiency rectification and values of $S_{11}$ parameter less than $-10 \mathrm{~dB}$ (which ensures a good impedance matching between rectifier and antenna). The results obtained after optimization process, presented in Table II, reached the established requirements and the optimized dimensions were used to fabricate a rectifier prototype, which was experimentally evaluated by using the network analyzer Keysight ${ }^{\circledR}$ E5071C.

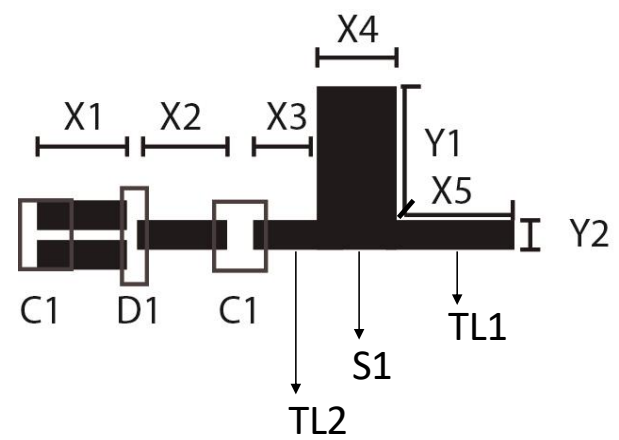

Fig. 5. Rectifier circuit topology.

TABLE II. RECTIFIER CIRCUIT GEOMETRICAL AND S11 PARAMETER

\begin{tabular}{cc}
\hline Rectifier parameters & Value \\
\hline X1 $(\mathrm{mm})$ & 5.000 \\
X2 $(\mathrm{mm})$ & 4.000 \\
X3 $(\mathrm{mm})$ & 3.000 \\
X4 $(\mathrm{mm})$ & 5.000 \\
X5 $(\mathrm{mm})$ & 13.000 \\
Y1 $(\mathrm{mm})$ & 11.000 \\
Y2 $(\mathrm{mm})$ & 2.000 \\
Simulated $\mathrm{S}_{11}(2.45 \mathrm{GHz})(\mathrm{dB})$ & -29.120 \\
Measured $\mathrm{S}_{11}(2.45 \mathrm{GHz})(\mathrm{dB})$ & -23.050 \\
\hline
\end{tabular}

The numerical and experimental results obtained, presented in Fig. 6, show good agreement and, again, the divergences are mainly due to glass non-homogeneity and anisotropy and to the manual manufacturing process.

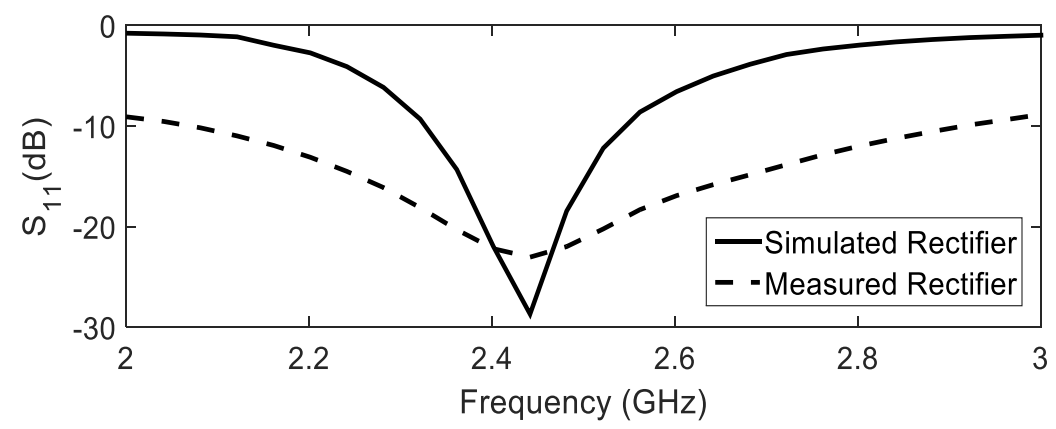

Fig.6. Simulated and measured rectifier $S_{11}$ parameter for rectifier circuit. 


\section{ELECTROMAGNETIC Window}

Aiming to increase the electromagnetic energy harvesting ability the electromagnetic window proposed in this work is composed of eight Single Rectennas (SR) mounted on the same glass substrate $(40 \times 30 \mathrm{~cm})$ with their DC output connected in parallel [8], as illustrated in Fig. 7. Each SR consists of a PAO-MTM and a rectifier circuit connected to each other. The spacing among SR (XR = $4.5 \mathrm{~cm}$ and $\mathrm{YR}=8 \mathrm{~cm}$ ) was designed by using the CST software in order to avoid electromagnetic interference among them and to maximize visibility through the window. The EW was used to feed a $\mathrm{RL}=5.2 \mathrm{~K} \Omega$ load once this is the EW output impedance value and it was chosen in order to match the load to EW.

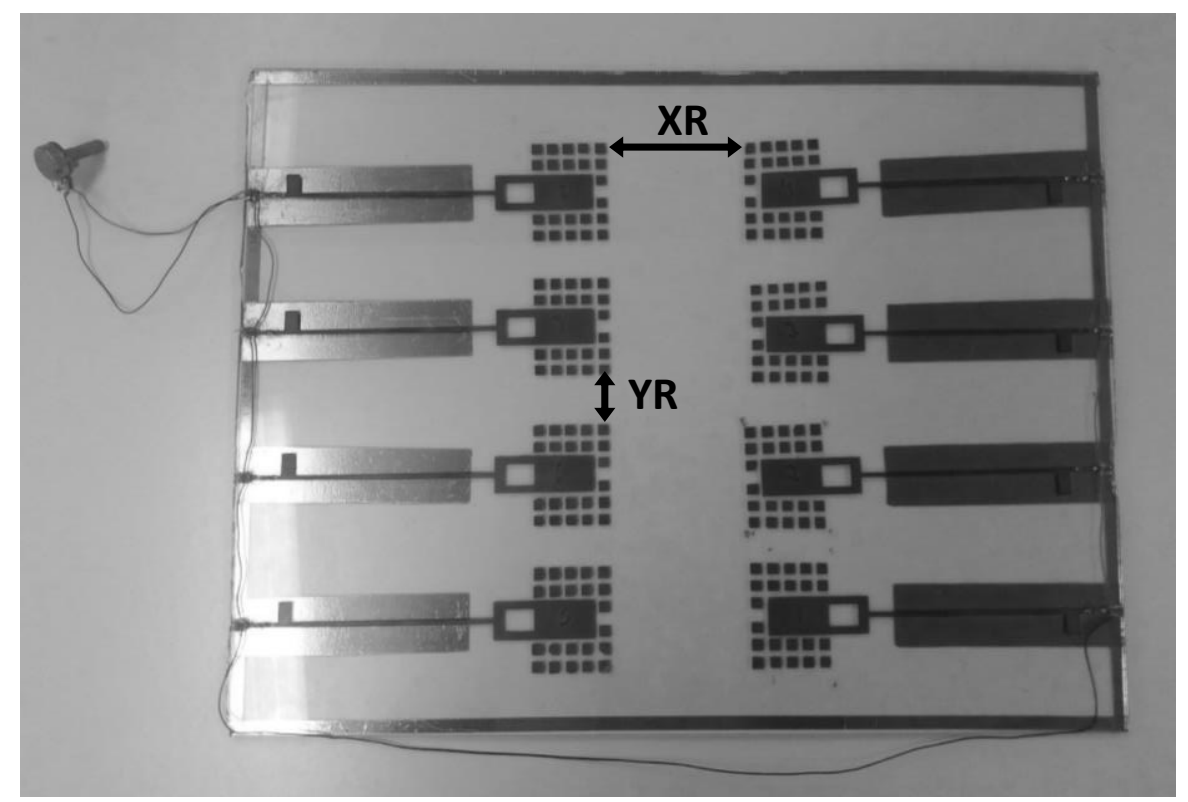

Fig. 7. Electromagnetic window (Prototype photo).

\section{RESULTS}

The performance of SR and EW was experimentally evaluated by using the setup illustrated in Fig. 8. A directive rectangular patch antenna, fabricated by using RT/duroid 5870 Roger substrate, was used as the transmitter antenna (TA) to provide the electromagnetic energy available in the environment. It was connected to the signal generator Keysight ${ }^{\circledR}$ N5181A adjusted to provide 18 $\mathrm{dBm}$ at $2.45 \mathrm{GHz}$ (18 dBm is internet Wi-Fi commercial router model TL-WR841HP output power). The distance d between TA and SR or EW was ranged from 5 to $100 \mathrm{~cm}$ and the open circuit voltage $\left(\mathrm{V}_{\text {open }}\right)$ and voltage $\mathrm{VL}$ across the $5.2 \mathrm{~K} \Omega$ resistor was measured by using the $2.5 \mathrm{GHz}$ oscilloscopic Keysight ${ }^{\circledR}$ DSOX6004A. The open circuit voltages obtained for the SR and the EW are shown in Fig. 9. As expected, the EW is able to provide a higher $\mathrm{V}_{\text {open }}$ value for longer distances. However, for small distances SR provides larger $\mathrm{V}_{\text {open }}$ values since it was placed perfectly aligned with TA and at the same distances, similar alignment was not reached by any SR element in the EW. The output power was evaluated by using $\mathrm{Po}=\mathrm{VL}^{2} / 5.2 \mathrm{~K} \Omega$ and the results obtained for the $\mathrm{EW}$ and for the same EW without MTM cells (EW-PAO) are presented in Fig. 10. 


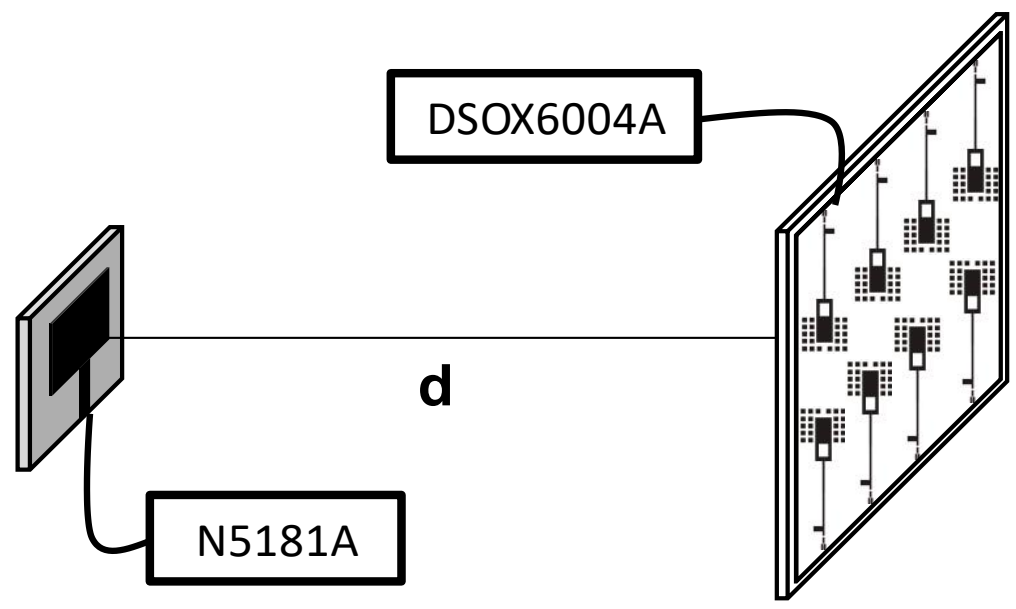

Fig. 8. Measurement setup.

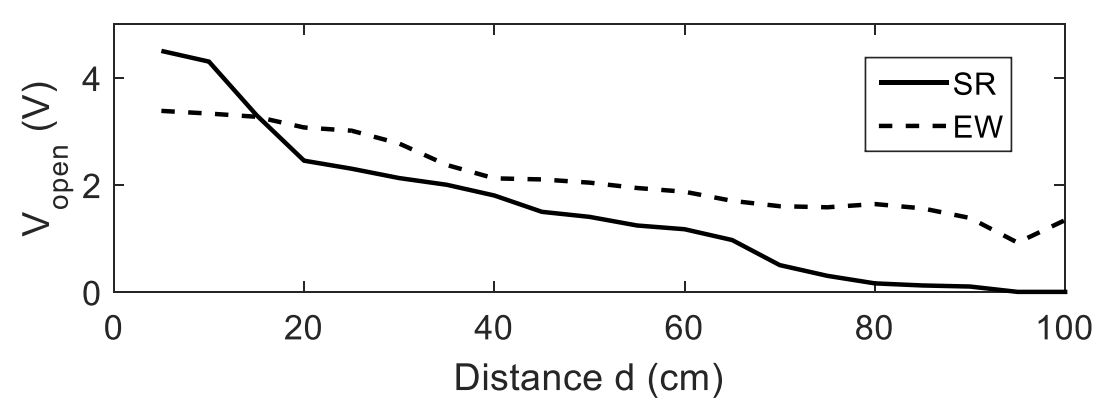

Fig. 9. Measured open circuit voltage x d.

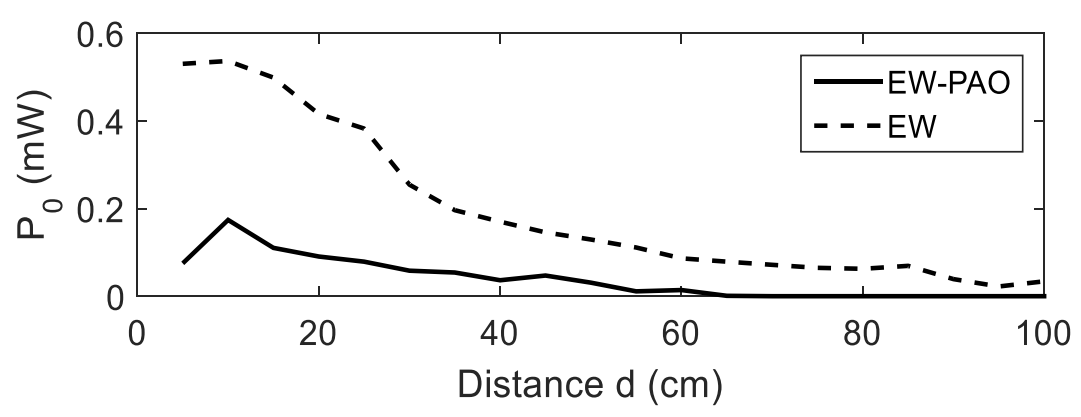

Fig. 10. Measured output power x d.

As it can be observed, the use of MTM cells leads to a significant increase in the electromagnetic energy harvesting ability. Po values achieved by EW (from 0.045 to $0.56 \mathrm{~mW}$ ) are sufficient, by employing an intermittent process, to feed WSNs [3]. For comparison purposes and in order to demonstrate the robustness of the proposed EW, the rectenna presented in [13], which is built on a transparent Plexiglas substrate, is able to provide values of power in the range from 1 to $5000 \mu \mathrm{W}$.

From these obtained results it is possible conclude that a greater amount of power can be harvested if several connected EWs are used in entire building's facade. The measured EW efficiency ( $\eta=$ $\mathrm{Po} / 18 \mathrm{dBm}$ ) for $\mathrm{d}=40 \mathrm{~cm}$ and different values of RL is presented in In Fig. 11. As expected, the highest efficiency value is reached for $\mathrm{RL}=5.2 \mathrm{~K} \Omega$, when EW and RL impedances are matched. The $\eta$ low values found are expected since the TA radiates electromagnetic energy in others directions that Brazilian Microwave and Optoelectronics Society-SBMO received 13 Sept 2019; for review 19 Sept 2019; accepted 24 Nov 2019 Brazilian Society of Electromagnetism-SBMag 
is not captured by the rectenna.

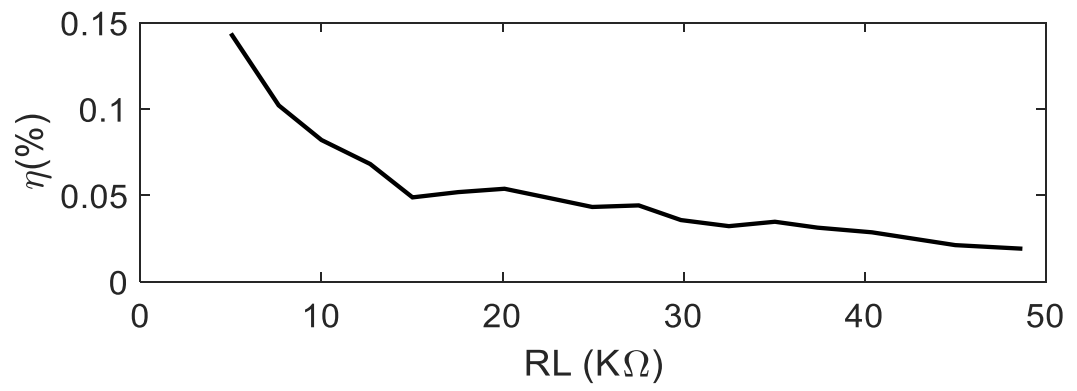

Fig. 11. Measured efficiency x RL.

\section{CONCLUSION}

In this work, a new rectenna constructive model and application for electromagnetic energy harvesting were proposed. The developed system operates at $2.45 \mathrm{GHz}$ harvesting the energy from the environment, it is visually appropriate to cover the large buildings facade and it can supply low power consumption loads. It has been demonstrated that the employment of more than one rectenna connected in parallel and the use of MTM structures provide an expressive increase of electromagnetic energy harvested [6].

\section{ACKNOWLEDGMENT}

This work was supported by the Brazilian Agencies: Conselho Nacional de Desenvolvimento Científico e Tecnológico (CNPq), Fundação de Amparo à Pesquisa de MG (FAPEMIG), Coordenação de Aperfeiçoamento de Pessoal de Nível Superior (CAPES), Centro Federal de Educação Tecnológica de Minas Gerais (CEFET-MG).

\section{REFERENCES}

[1] W. C. Brown, "The history of power transmission by radio waves," IEEE Trans. Microwave Theory Tech. 32, pp. 1230-1242, Sep. 1984.

[2] B. Essakhi, L. Pichon, and G. Akoun, "Fast analysis of a broad-band microwave rectenna using 3-D FEM and Padé approximation," IEEE Trans. on Magnetics, vol. 43, no. 4, pp. 1309-1312, Apr. 2007.

[3] K. Shafique, B. Khawaja, M. Khurram, S. Sibtain, Y. Siddiqui, M. Mustaqim, H. Chattha, and X. Yang, "Energy harvesting using a low-cost rectenna for Internet of things (IoT) applications," IEEE Access, May 2018.

[4] P. Thosar, and R. Mathur, "Design of rectenna using RF harvesting for batteryless IoT sensors," International Journal on Future Revolution in Computer Science \& Communication Engineering (IJFRSCE), vol. 4, no. 3, pp. 63-67, Mar. 2018.

[5] E. M. Ali, N. Z. Yahaya, N. Perumal and M. A. Zakariya, "Design and development of harvester rectenna at GSM band for battery charging applications," ARPN Journal of Engineering and Applied Sciences, vol. 10, no. 21, Nov. 2015.

[6] H. Takhedmit, Z. Saddi, and Laurent Cirio, "A high-performance circularly-polarized rectenna for wireless energy harvesting at 1.85 and $2.45 \mathrm{GHz}$ frequency bands," Progress In Electromagnetics Research C, vol. 79, pp. 89-100, 2017.

[7] A. Hamani, B. Allard, T. P. Vuong, M. C. E. Yagoub, and R. Touhami, "Design of rectenna series-association circuits for radio frequency energy harvesting in CMOS FD-SOI 28 nm," IET circuits, Devices \& Systems, vol 12, pp. 40-49, Jan 2018.

[8] N. Degrenne, V. Marian, C. Vollaire, F. Buret, J. Verdier, and B. Allard, "Voltage reversal in unbalanced rectenna association,” IEEE Antennas Wireless Propagat. Lett., vol. 11, pp. 941-944, Aug. 2012.

[9] V. Palazzi, J. Hester, J. Bito, F. Alimenti, C. Kalialakis, A. Collado, P. Mezzanotte, A. Georgiadis, L. Roselli, and M. Tentzeris, "A novel ultra-lightweight multiband rectenna on paper for RF energy harvesting in the next generation LTE bands," IEEE Trans. Microw. Theory Techn., vol. 66, no. 1, pp. 366-379, Jan. 2018. 
[10] W. Cao, B. Zhang, J. Jin, W. Zhong, and W. Hong, "Microstrip antenna with electrically large property based on Metamaterial inclusions," IEEE Trans. Antennas and Propag., vol. 65, no. 6, pp. 2899-2905, Jun. 2017.

[11] V. G. Veselago, "The electrodynamics of substances with simultaneously negative values of permittivity and permeability," Soviet Phys. Uspekhi, vol. 10, no. 4, pp. 509-514, 1968.

[12] R. Rani, P. Kaur and N. Verna, "Metamaterials and Their Applications in Patch Antenna: A Review," International Journal of Hybrid Information Technology, vol. 8, no.11, pp. 199-212, 2015.

[13] S. Bellal, H. Takhedmit and L. Cirio, "Design and experiments of transparent rectennas for wireless power harvesting," IEEE Wireless Power Transfer Conference WPTC'2016, Aveiro, Portugal, May 2016. 